\title{
Translation of Research Into Practice: Why We Can't "Just Do It"
}

\author{
Lee A. Green, MD, MPH, and Colleen M. Seifert, $P h D$
}

Translation of new knowledge into practice proceeds through 3 stages, from awareness through acceptance to adoption. Translational research focuses almost exclusively on the first 2 stages. We argue that improving the disappointing results of translation efforts will require a detailed understanding of how adoption takes place. We summarize research in cognitive science that illustrates how accepted "declarative" knowledge (acquired through lectures, reading, and discussion) differs (even down to its locus in the brain) from adopted "procedural" knowledge that is acted on in clinical practice. We suggest strategies that can capitalize on the cognitive processes by which declarative knowledge is proceduralized, as a means of making translation more effective, including (1) structured case-level feedback, automated or from human consultants, during the declarative stage; (2) practice in context early in the procedural stage; and (3) deliberative practice when procedural knowledge has been formed but is still being refined. (J Am Board Fam Pract 2005;18:541-5.)

The problem of translating research into practice has come sharply into focus over the past several years. ${ }^{1}$ Many evidence-based practice guidelines have been developed over the last decade with the aim of improving the use of interventions of proven benefit for patient-oriented outcomes. A wide variety of individual interventions has been attempted, including at least 6 categories identified by Eisenberg ${ }^{2}$ : education, positive or negative feedback, positive or negative incentives, and administrative regulations and sanctions. However, the generally very modest effects these methods have achieved $^{3}$ have led to widespread agreement that physicians and health care systems simply do not put new knowledge about how to improve our patients' outcomes into practice nearly quickly enough. $^{4-7}$

Translation of research into clinical practice is often conceptualized as proceeding from awareness through acceptance to adoption. ${ }^{8}$ Educational meth-

Submitted 9 November 2004; revised 4 July 2005; accepted 7 July 2005.

From the University of Michigan, LS\&A Psychology Department (CMS), and Department of Family Medicine (LAG), Ann Arbor, MI.

Conflict of interest: none declared.

Corresponding author: Lee Green, MD, MPH, Associate Professor, University of Michigan, Department of Family Medicine, 1018 Fuller, Ann Arbor, MI 48109-0708 (e-mail: greenla@umich.edu). ods, such as disseminating practice guidelines and continuing medical education, clearly aim at awareness and acceptance. But although there has been a great deal of research identifying factors that affect adoption, there is little understanding of how adoption actually takes place. The physician begins with a great deal of prior knowledge, and it is into this well-oiled machinery that the physician attempts to integrate new evidence from a journal report, continuing education course, or practice guideline. For example, consider the guideline that "congestive heart failure patients should be evaluated for use of beta-blockers." An expert physician may be aware of this recommendation and may wholeheartedly accept it as good practice, but may still fail to adopt it when they happen to see an elderly patient in the clinic who could benefit from beta-blockade. Knowledge of evidence can remain separate from, and not integrated into, the physician's extensive database of procedures that guides their decisions and actions. This makes the likelihood of recognizing that the new knowledge is appropriate and incorporating it into these well-rehearsed procedures very uncertain.

How do physicians translate new knowledge into specific clinical actions that they adopt into practice? Currently, the physician is left on her own to "just do it": to figure out when and how often a relevant case may appear in their practice, what a 
target case will look like in presentation, how to incorporate the evidence into a change in their practices, how to handle inconsistencies in evidence tools (eg, Up-To-Date) and other common practices. Further, physicians are expected to accomplish these changes within an already overcrowded, demanding clinic schedule. The very modest results of practice change research to date ${ }^{1}$ argue that this approach is, at best, unrealistic. In this article, we suggest that understanding the adoption process requires considering the basic science of human cognition in primary care. By examining the cognitive processes involved in expert learning, we identify key features of the successful integration of new into existing knowledge and suggest ways to support physicians in the translation of new knowledge into practice.

\section{Cognitive Processes in the Clinic}

The cognitive challenge faced by the busy primary care clinician is to translate new explicit knowledge into well-rehearsed procedures that can be executed almost automatically. Studies of cognitive skill learning have identified a central shift in how knowledge is represented as the learner goes from novice to expert. The new information is first represented in explicit, declarative form, but is gradually transformed with experience to a more implicit procedural form. ${ }^{9-13}$ This mode shift is not a trivial process; in fact, research has shown that declarative and procedural knowledge are stored in separate areas within the brain. ${ }^{14} \mathrm{~A}$ general interpretation of these results is that new explicit memories are formed in the hippocampus, ${ }^{15}$ but with experience, this information is distributed in the cortex. ${ }^{16}$ The available evidence is compelling that procedural learning involves structures different from those involved in explicit declarative learning. ${ }^{17}$

Thus, for successful adoption to take place, physicians must shift their new knowledge from the explicit declarative information they are given into a procedural mode, where the knowledge is encapsulated into well-rehearsed procedural rules. Early work on skill learning ${ }^{1-12}$ proposed this mode shift as the first of 3 developmental stages in skill acquisition:

1. Declarative stage: construct a memory representation of new information.

2. Procedural stage: translate declarative knowledge into action rules: IF_THEN_.
3. Autonomous stage: rehearse procedural rules to function automatically.

Anderson further developed this stage model and demonstrated its success in predicting in detail how performance speeds up with practice, how errors are corrected, and how expertise becomes, in a sense, automatic. ${ }^{13,18,19}$ In the first stage, called the declarative stage, ${ }^{13}$ the learner receives instruction and constructs an understanding of the facts. Declarative knowledge is what we typically mean when we refer informally to factual knowledge, assessed on traditional academic tests. Existing efforts in research translation, including medical continuing education, practice guidelines, research reports, and on-line evidence access, seem to successfully promote the construction of new declarative knowledge among physicians. ${ }^{3}$ In addition, because quickly acquiring and remembering information is a skill that medical schools select for, physicians are quite adept at grasping complex reports and encoding them into memory. However, an expert physician seems to reason only rarely using declarative knowledge, because it is simply too time-consuming and effortful, especially within the time demands of the clinic schedule. In addition, reasoning from "first principles" can be hazardous without carefully considering information about its actual utility in the environment. The transition from declarative knowledge to the use of that knowledge may be where our current translation efforts fall short.

Instead, the actual practice of primary care, like expert behavior in other settings, ${ }^{20}$ draws largely on procedural knowledge (sometimes termed "knowledge in action"). This second stage involves transforming the knowledge from declarative form into procedural form, so that it can be directly acted on without further interpretation. ${ }^{15,18}$ Procedural rules take the form "if $\rightarrow$ then," where the "if" consists of the conditions for applying the new rule, and the "then" directs specific actions to take. For example, given new practice guidelines for heart failure care, the learner should proceduralize the recommendations into the following form: "If the diagnosis is congestive heart failure, then consider prescribing beta-blockers." Procedural rules specify conditions to watch for in the clinical setting, and when matched, indicate specific actions to follow.

The third stage, called the autonomous stage, ${ }^{12}$ involves gradual, continued improvement in the 
performance of the intended skill. Anderson notes this stage requires practice using the knowledge appropriately and results in a gradual speedup in execution. ${ }^{13}$ By this stage, the cognitive process simplifies to one of pattern matching, where the expert makes rapid efficient decisions based on past patterns of features. ${ }^{9,10,19}$ Experienced primary care clinicians, after years of practice, possess rich and diverse sets of well-encoded procedural rules. Expert physicians can make decisions using these highly practiced patterns quickly and seamlessly and with minimal deliberate thought.

\section{Translating New Knowledge into Action}

Research on human cognition has demonstrated that overcoming well-practiced rules to incorporate new information is one of the great challenges in learning. ${ }^{13}$ However, these cognitive processes have been largely ignored in medical translational research to date. How might we do better? The answers to that question are crucial to translational research, guideline implementation, quality improvement, and continuing medical education; indeed, to all attempts to change physician practices. Based on the 3-stage model of skill acquisition and related research, we propose some principles to guide in the design of training for evidence-based medicine.

\section{Declarative Stage: Debugging Knowledge through Feedback}

A key feature of learning during the declarative stage is debugging or elaborating, testing, and correcting the new knowledge. During medical school, support for knowledge debugging is built in through the apprenticeship model of medical training. Learners report to and operate under domain experts who guide them in implementing the declarative knowledge into procedural form. However, current approaches to updating physician knowledge include no method of determining the outcome of the learners' efforts. There is no guidance or evaluation to assist physicians in recognizing when they are failing to apply their new knowledge and no feedback on how to improve their performance.

Some current approaches to translation have produced methods that can assist clinicians in changing practices. For example, real-time clinical reminder systems ${ }^{21}$ can serve to highlight opportunities to apply new information. However, current systems are not designed to reinforce the new knowledge, nor are they tuned to the physician's performance. As a result, they are often actively ignored and as prone to disrupt clinicians' rulebased decision processes as to facilitate them. ${ }^{22}$ One possible extension to clinical prompting systems is to tailor the reminders to individual physicians' learning goals. So, for example, on-line evidence sources could be tied into prompting systems, so that physicians could add new reminders to their personal portfolio as they review evidence, and these new reminders could be added to reminders for future cases seen in clinic.

Another approach is to introduce a "consultation" format following continuing education courses. As a "new practices" follow up, instructors could contact physicians by phone or e-mail and ask about recent cases (for example, of congestive heart failure) seen in clinic. Then a discussion of practices in considering beta-blockers could occur. Or noninteractive "self tests" could ask learners to think about recent cases and whether they used specific practices. Even if physicians choose not to fully disclose their actions within a conversation, the consultation serves to model a process of debugging use of new knowledge. Much more research will be needed to understand how to provide helpful feedback as physicians practice their adapted procedures in the primary care office setting.

\section{Procedural Stage: Practicing New Rules in Context}

A key feature of the procedural stage is the development of associations among related rules. Even when a correct set of procedural rules is developed, the execution of the rules will not take place in a vacuum, but instead in the presence of many other rules. The newly acquired rule competes with existing rules, and associations among rules as they are actually used in practice soon develop. ${ }^{13}$ With more experience, the new rule becomes embedded into a network of associations among rules that co-occur, which helps to ensure the best rule is accessed for a given situation. Further, incorporating the new rule may require shifting the hard way, from rule-based to an explicit declarative mode to examine the relationships among the rules. The new evidence may connect with existing rules in memory in a variety of ways: it may contradict existing rules (eg, the pre-1990s teaching of "never 
use beta-blockers in congestive heart failure patients"), interact with existing rules ("check for comorbid conditions that may contraindicate betablockers"), or require further discrimination among existing rules ("congestive heart failure in elderly versus younger patients"). Based on learning research, the use of the new rules must be practiced in the actual context where they are needed. ${ }^{23,24}$

Current methods introduce new evidence when a physician is out of the clinic context; for example, reviewing research findings, reading about new practice guidelines, consulting with colleagues, or taking a continuing medical education course. In fact, physicians are most likely to become aware of and to accept new knowledge in any setting except the one in which they need to apply it: namely, the clinic visit. A wealth of cognitive research demonstrates that the greater the commonality between learning context and application context, the greater the likelihood that new information will be spontaneously applied. ${ }^{23,24}$ The American Board of Family Medicine's new Maintenance of Certification program is a step in this direction, because it replaces paper-and-pencil tests of purely declarative knowledge with interactive case-based learning and testing modules.

As is the case with mode shift, context shift is not a trivial process. Hence, much remains to be done to develop and deploy methods of reinforcing and practicing new knowledge within the context of its application. One approach is to develop more realistic primary care case simulations (current classroom problem-based learning approaches may approximate the clinical context quite poorly ${ }^{25}$ ). Based on theories of human learning, simulations should include features such as exposure to a series of related cases, guided instruction for initial rule application, structured or "scaffolded" lessons, and especially, making use of the contextual cues present in the clinic visit, including multiple medical problems, patient psychosocial concerns, and physical setting and administrative details. The new knowledge must be incorporated within the messy set of competing associations that actually occur in the clinic context.

\section{Autonomous Stage: Learning to Practice "Deliberately"}

The experience of using new rules in a variety of cases is critical for the development of fluency in practice, the third stage of skill acquisition. For example, a new rule may not be encoded with the most appropriate conditions, so it may occasionally be accessed when it is in fact not needed. ${ }^{25}$ This process of respecifying the conditions for applying the rule, called tuning, may require extensive practice in varied instances before the rule is reliably matched only when appropriate. ${ }^{13}$ Compounding the problem is that, unlike in specialty practice, the primary care patient is unlikely to have the same problem as the last patient, so an entirely different set of rules may be accessed every few minutes. Lapses in exposure to similar cases may prohibit any opportunity to practice the new knowledge several times in succession.

In current continuing medical education training, practice with example cases is sometimes provided through paper or video cases that illustrate the lesson. However, distributed practice with the material should also be provided that replicates the experience of the physician when in the clinic. For example, practice with a set of congestive heart failure cases will help to develop new procedures, but the real task is to access those procedures from a setting where none of the recent cases are related. One proposal is to provide a large case database where physicians can test themselves on selected types of cases, or choose a new randomized case set with links to recent best-practice guidelines. Use of the database self-test could be tied to continuing education credit, or simply provided as a resource for the physician. The ability to retrieve the appropriate procedural rules in competition with other possible rules is a key part of learning when to best apply a new rule.

Cognitive skill research shows that elite performers across domains (including musicians, chess experts, performance arts, and sports) require about 10 years of intense preparation. ${ }^{10}$ This suggests the time scale of required attention to changes in knowledge should be lengthened. One continuing education course on new practices will not provide adequate practice in using the procedural knowledge; instead, an ongoing series of interventions, reminders, and evaluations will be necessary as experience with cases accumulates for an individual physician within her practice. A key element of those who go on to attain expert performance is "deliberate practice," where specific aspects of performance are addressed through thoughtful repetition and successive refinement. ${ }^{26}$ Further research is needed on how some individual physicians de- 
velop learning goals and deliberately practice to achieve success in incorporating new evidence.

\section{Conclusion}

We have proposed guidelines for the translation of new knowledge into practice that are based on empirical findings on the cognitive processes involved in expert learning. Obviously, embedding supports for cognitive change into practice has the potential to disrupt current practices in the timepressured environment of general medicine. However, any loss of efficiency that arises from supporting physicians as they adopt new guidelines will be offset by the gains in efficiency from their evidence-based practice. It is the accumulated evidence that provides the strongest criterion for the success of medical practice; so, to achieve the best outcomes for patients, it is critical to build in new ways to support expert physicians as they adopt changes in practice.

\section{References}

1. Lenfant C. Shattuck lecture-clinical research to clinical practice-lost in translation? N Engl J Med 2003;349:868-74.

2. Eisenberg JM. Doctors' decisions and the cost of medical care: the reasons for doctors' practice patterns and ways to change them. Ann Arbor (MI): Health Administration Press; 1986.

3. Oxman AD, Thomson MA, Davis DA, Haynes RB. No magic bullets: a systematic review of 102 trials of interventions to improve professional practice. CMAJ 1995;153:1423-31.

4. Specialty care: heart attack survivors treated by cardiologists more likely to take recommended drugs: United States General Accounting Office; 1998. Pub. No. GAO/HEHS-99-6.

5. Schuster MA, McGlynn EA, Brook RH. How good is the quality of health care in the United States? Milbank Q 1998;76:517-63, 509.

6. McGlynn EA, Asch SM, Adams J, et al. The quality of health care delivered to adults in the United States. N Engl J Med 2003;348:2635-45.

7. Grol R, Grimshaw J. Evidence-based implementation of evidence-based medicine. Jt Comm J Qual Improv 1999;25:503-13.

8. Davis DA, Taylor-Vaisey A. Translating guidelines into practice. A systematic review of theoretic concepts, practical experience, and research evidence in the adoption of clinical practice guidelines. CMAJ 1997;157:408-16.

9. Simon HA, Chase WG. Skill in chess. Am Sci 1973; 61:394-403.

10. Ericsson KA., Charness, N. Expert performance: its structure and acquisition. American Psychologist 1994:49:725-47.

11. Fitts PM. Perceptual motor skill learning. In: Melton AW, ed. Categories of human learning. New York: Academic Press; 1964.

12. Fitts PM, Posner MI. Human performance. Belmont (CA); 1967.

13. Anderson JR. Acquisition of cognitive skill. Psychological Review 1982;89:369-406.

14. Squire LR, Zola SM. Structure and function of declarative and nondeclarative memory systems. Proc Natl Acad Sci U S A 1996;93:13515-22.

15. Schacter DL, Badgaiyan, RD. Neuroimaging of priming: new perspectives on implicit and explicit memory. Curr Dir Psychol Sci 2001;10:1-4.

16. Koutstaal W, Wagner AD, Rotte M, Maril A, Buckner RL, Schacter DK. Perceptual specificity in visual object priming: fMRI evidence for a laterality difference in fusiform cortex. Neuropsychologia 2001;39: 184-99.

17. Anderson JR. Cognitive psychology and its implications. NewYork: Worth Publishers; 2005.

18. Anderson J. ACT: A simple theory of complex cognition. Am Psych 1996;51:355-65.

19. Anderson JR. Rules of the mind. Hillsdale (NJ): Lawrence Erlbaum Associates; 1993.

20. Zsambok CE, Klein G. Naturalistic decision making. Mahwah (NJ): Lawrence Erlbaum Asociates; 1997.

21. Balas EA, Weingarten S, Garb CT, Blumenthal D, Boren SA, Brown GD. Improving preventive care by prompting physicians. Arch Intern Med 2000;160: 301-8.

22. Hunt DL, Haynes RB, Hanna SE, Smith K. Effects of computer-based clinical decision support systems on physician performance and patient outcomes: a systematic review. JAMA 1998;280:1339-46.

23. Tulving E, Thomson DM. Encoding specificity and retrieval processes in episodic memory. Psychol Rev 1973;80:352-73.

24. Patalano AL, Seifert CM. Opportunistic planning: being reminded of pending goals. Cognit Psychol 1997;34:1-36.

25. Vanderstoep SW, Seifert CM. Learning 'how' vs. learning 'when': improving problem solving transfer. J Learn Sciences 1993;3:93-111.

26. Ericsson KA, Krample RT, Tesch-Romer C. The role of deliberate practice in the acquisition of expert performance. Psych Rev 1993;100:363-406. 